\title{
O IMPACTO DA ASMA, DA RINITE ALÉRGICA E DA RESPIRAÇÃO ORAL NA QUALIDADE DE VIDA DE CRIANÇAS E ADOLESCENTES
}

\section{Impact of asthma, allergic rhinitis and mouth breathing in life quality of children and adolescents}

\author{
Silvia Márcia Andrade Campanha (1), Lincoln Marcelo Silveira Freire (2), \\ Maria Jussara Fernandes Fontes ${ }^{(3)}$
}

\begin{abstract}
RESUMO
Tema: qualidade de vida em pacientes com doenças respiratórias. Objetivo: rever a literatura enfocando o impacto da asma, da rinite alérgica e da respiração oral na qualidade de vida do paciente, as relações entre essas morbidades e medidas que possam diminuir esse impacto. Conclusão: há consenso na literatura de que existe inter-relação dessas co-morbidades. O impacto da asma, rinite alérgica e respiração oral afetam diretamente a qualidade de vida do indivíduo não só pela alteração respiratória, mas, também pelos prejuízos comportamentais, funcionais e físicos que ocasionam. $O$ controle dessas morbidades é um tema usual na literatura. Novas propostas terapêuticas englobam o envolvimento de equipes multiprofissionais. Há programas de saúde estruturados com essa visão e objetivam não só melhorar a condição respiratória, mas, também propõem medidas que analisam a qualidade de vida e possibilitam avaliação global do paciente.
\end{abstract}

DESCRITORES: Asma; Rinite; Respiração Bucal; Perfil de Impacto da Doença; Qualidade de Vida

\section{INTRODUÇÃO}

A asma e a rinite alérgica apresentam elevada prevalência na população pediátrica. A prevalência da rinite alérgica foi referida nas maiores cidades

(1) Fonoaudióloga; Professora da Graduação de Fonoaudiologia da Faculdade de Estudos Administrativos - Centro de Gestão Empreendedora; Especialista em voz pelo CEFAC Saúde e Educação; Especializanda em Motricidade Orofacial pelo CEFAC - Saúde e Educação; Mestre em Ciências da Saúde do Instituto de Previdência dos Servidores do Estado de Minas Gerais.

(2) Pediatra; Presidente da Academia Mineira de Pediatria; Membro da Academia Brasileira de Pediatria; Membro do colegiado de pós-graduação Universidade Federal de Minas Gerais e Instituto de Previdência dos Servidores do Estado de Minas Gerais; Professor Associado Doutor do Departamento de Pediatria da Faculdade de Medicina da Universidade Federal de Minas Gerais.

(3) Pediatra; Professora de Pediatria e Pneumologia Pediátrica da Faculdade de Medicina da Universidade Federal de Minas Gerais; Professora da Pós-Graduação em Ciências da Saúde da Criança e do Adolescente da Faculdade de Medicina da Universidade Federal de Minas Gerais; Doutora pelo Departamento de Pediatria da Faculdade de Medicina da Universidade Federal de Minas Gerais. do Brasil e atingiu até $31,7 \%$ em crianças de sete a 14 anos, superior a ocorrência de asma na população humana ${ }^{1}$. Representa, portanto, um problema global de saúde pública que atinge no mínimo, 10 a $25 \%$ da população geral ${ }^{2}$. Dados do International Study of Asthma and Allergies in Childhood revelam valores sobre a prevalência da asma que oscilou, na faixa etária de seis a sete anos, entre $4,7 \%$ e $20,7 \%$ e na faixa etária de 13 a 14 anos, entre 4,8 e $21,9 \%$. Considerando as duas populações, a prevalência cumulativa média foi de $13,3 \%{ }^{3}$. No que se refere à hospitalização dos pacientes asmáticos, em 1996, foi considerada a quarta causa de internação pelo Sistema Único de Saúde (SUS), sendo, portanto, um problema de saúde pública. Estudos realizados no Rio Grande do Sul revelam que a prevalência da asma dobrou no período de 12 anos em escolares ${ }^{4}$.

No final da década de 90, o International Study of Asthma and Allergies in Childhood revelou que a comorbidade entre asma e rinite alérgica pode alcançar até $80 \%{ }^{3}$. Aspectos morfológicos e fisiológicos revelam as semelhanças e as diferenças estruturais entre a mucosa nasal e brônquica e entre os meca- 
nismos que explicam a influência da rinite alérgica na asma, incluindo a influência da respiração oral secundária à obstrução nasal ${ }^{5}$. A respiração oral, apesar de ser um sintoma, pode ser considerada uma síndrome, pois incorpora vários sintomas como alterações orofaciais, posturais, oclusais e distúrbios de comportamento ${ }^{6}$. Há casos que apresentam ainda alteração na capacidade pulmonar, diminuição do pico de fluxo expiratório e da expansibilidade torácica ${ }^{7}$ além da dificuldade inspiratória usual. A interação asma, rinite alérgica e respiração oral já está bem estabelecida, promovendo alteração no crescimento facial e somático, visualizadas, sobretudo na infância ${ }^{8}$ e o seu impacto com evolução crônica reflete na vida emocional, física e social do indivíduo ${ }^{9}$.

Atualmente, utilizam-se critérios de avaliação para asma e rinite alérgica de maneira ampla e integral, considerando não somente a terapêutica, mas também o complexo indivíduo, doença e suas inter-relações ${ }^{9}$. Decorrente da diversificação dos sintomas da respiração oral e sua interação com a asma, o tratamento do paciente asmático com rinite alérgica e respiração oral requer uma equipe multiprofissional (médicos, odontopediatras, ortodontistas, fisioterapeutas e fonoaudiólogos) ${ }^{8}$. A abordagem profilática dessas morbidades reduz hospitalizações e atendimentos de urgência, fato comprovado na literatura internacional ${ }^{10}$ e também em nosso meio, refletindo em melhoria da qualidade de vida ${ }^{11}$.

Devido à elevada prevalência na população humana e ao grande impacto dessas doenças crônicas na qualidade de vida do indivíduo, objetivase apresentar neste artigo de revisão o impacto da asma, da rinite alérgica e da respiração oral na qualidade de vida. Objetiva ainda relatar medidas que diminuem o impacto causado por essas co-morbidades, relatando também as suas inter-relações.

\section{MÉTODOS}

O levantamento bibliográfico foi realizado a partir das informações disponibilizadas por livros, teses, dissertações, artigos de bases de dados LILACS e MEDLINE nos últimos vinte anos, utilizando os termos: impacto da asma e/ou da rinite alérgica e/ou da respiração oral na qualidade de vida. Os descritores utilizados foram asma, rinite, respiração bucal, perfil de impacto da doença, qualidade de vida. Foram destacados 35 estudos relevantes, recentes, com metodologia considerada adequada que enfatizavam a inter-relação das morbidades estudadas e o impacto da asma, rinite alérgica e respiração oral na qualidade de vida de crianças e adolescentes.
Os achados na literatura foram apresentados e posteriormente discutidos.

\section{REVISÃO DA LITERATURA}

A asma caracteriza-se principalmente pela presença de obstrução do fluxo aéreo e de fenômenos como hiper-responsividade e inflamação crônica das vias aéreas ${ }^{12}$. Considerada uma doença crônica geralmente reversível gera sofrimento a pacientes e familiares, evoluindo eventualmente para o óbito. Nos últimos anos, tem sido registrado aumento da incidência de morbidade e mortalidade da asma ${ }^{4}$.

As dificuldades no seu manejo persistem, apesar da disponibilidade de recursos terapêuticos eficazes. E a ausência do acompanhamento médico e de tratamento preventivo também contribui para a ocorrência dos atendimentos de urgência e hospitalizações ${ }^{12}$.

Num passado recente, a terapêutica da asma se limitava ao uso de broncodilatadores e da corticoterapia em altas doses para reverter as agudizações. Enquanto, medidas profiláticas investiam apenas no controle ambiental ${ }^{12}$.

Nos últimos anos foram observados grandes avanços na compreensão da fisiopatogenia da asma bem como em relação aos recursos terapêuticos. Estabeleceu-se a compreensão de que a patogenia básica da asma é eminentemente inflamatória. Paralelamente, a via inalatória passou a ser utilizada na administração de drogas necessárias ao controle dessa inflamação, tanto nos períodos de agudização como nos períodos intercrise. Surgiram novas drogas e vários centros passaram a realizar provas de função pulmonar ${ }^{12}$. Enfim, há uma busca constante em melhorar a qualidade de vida dos pacientes com asma ${ }^{13}$.

Em crianças asmáticas, a medida de qualidade de vida foi por muito tempo baseada na medida convencional da gravidade da asma, na presença e na intensidade dos sintomas, na necessidade de medicação e de discussão com os pais e em alguns centros na mensuração da função pulmonar ${ }^{14}$. No entanto, atualmente, há clara evidência de que os parâmetros clínicos têm fraca relação com o que a criança está sentindo e como estão suas funções diárias ${ }^{14}$.

A partir da análise de dados epidemiológicos, de custos e da avaliação dos aspectos sociais e psicológicos envolvidos na asma, levantou-se a hipótese de que a avaliação clínica dessa doença, de fato, enfoca uma só faceta do grande impacto que ela desempenha na vida do paciente e na sociedade. Assim, a medida da qualidade de vida tem papel importante no tratamento e acompanhamento dos pacientes com asma, e cada vez mais 
o termo qualidade de vida torna-se um marco na vida diária do indivíduo ${ }^{15}$.

A rinite alérgica é caracterizada por uma inflamação das membranas nasais, induzida por exposição a alérgenos ${ }^{2}$. Clinicamente, a rinite alérgica é caracterizada por prurido nasal intenso, espirros em salva, obstrução nasal, coriza hialina, respiração oral, diminuição de olfato, sintomas decorrentes do processo inflamatório ${ }^{2,16}$.

Profissionais especializados em cuidados de pacientes com rinite alérgica focam as orientações sobre o controle ambiental, o tratamento medicamentoso e a imunoterapia ${ }^{\text {? }}$.

Pacientes com rinopatia alérgica podem apresentar déficit cognitivo relacionado ao clássico uso dos anti-histamínicos que ao atravessarem a barreira hematoencefálica aumentam a sonolência que advém do sono insatisfatório decorrente da obstrução nasal ${ }^{17}$.

O cansaço provocado pela congestão nasal em pacientes alérgicos e a conseqüente respiração oral é um fator responsável pelos escores mais baixos de qualidade de vida apresentados por esses pacientes. A utilização de corticosteróides nasais tópicos reduz a congestão nasal e melhora a qualidade de sono e assim, diminui a sonolência diurna ${ }^{18}$.

Outro aspecto provém da condição crônica da doença que afeta também a qualidade de vida do indivíduo por longo tempo ${ }^{19}$. Esse fato toma maiores proporções diante da alta prevalência da rinite. Estudiosos alertam para o impacto negativo da doença sobre o aprendizado, a capacidade cognitiva, a memória, as relações psicossociais e também para os efeitos colaterais da terapêutica necessária ao seu controle. Destacam as alterações de comportamento decorrentes da rinite e sua responsabilidade na potencialização de sérias co-morbidades como a asma ${ }^{19,20}$.

O respirador oral é um indivíduo que possui uma capacidade respiratória nasal restrita, que o leva a respirar pela boca na maior parte do tempo. A respiração oral pode ser considerada uma síndrome, pois apresenta vários sintomas como alterações orofaciais, posturais, oclusais e distúrbios de comportamento ${ }^{6}$.

Em relação às alterações orofaciais a literatura cita a face longa e estreita; os lábios entreabertos ${ }^{21}$, com superior curto e inferior evertido; os maxilares pouco desenvolvidos; a cianose infraorbitária; o tônus da musculatura orofacial diminuído; a face pouco expressiva; a hipertonia do músculo mentalis; a hipotonia da língua; a deglutição atípica e as malformações craniofaciais ${ }^{22,23}$.

$\mathrm{Na}$ arcada dentária e na dentadura típica de um respirador oral é possível encontrar incisivos verticalizados, maxila atrésica com mordida cruzada, apinhamento dos incisivos e uma tendência à mordida aberta ${ }^{22}$. Autores descrevem sobre esses achados: alteração de oclusão, classe II de Angle (mandíbula encontra-se em posição mais distal em relação à maxila); palato ogival ${ }^{22,23 ;}$; hipodesenvolvimento da maxila e mandíbula, alteração na erupção dentária ${ }^{23}$.

Entre as alterações posturais observadas citamse a assimetria torácica, os ombros ântero-pulsionados, a cabeça fletida, a rotação dianteira dos ombros, o pescoço fletido para frente, a retificação da coluna cervical, o comprometimento da postura dos membros superiores e inferiores ${ }^{24}$.

Distúrbios de comportamento também são citados na literatura como a inquietação, a irritação, a desatenção, o sono agitado, a enurese noturna, a sonolência durante o dia. Esses sintomas podem afetar a concentração da criança e interferir negativamente no desempenho escolar ${ }^{25}$. Não é raro o respirador oral ter distúrbio de escolaridade apesar de inteligência normal ${ }^{24}$.

Diante do exposto é inquestionável a atuação negativa da respiração oral sobre a qualidade de vida do paciente. E, visando minimizar essas conseqüências da respiração oral na qualidade de vida do indivíduo, estudiosos relatam que o tratamento do respirador oral deve constar de uma equipe interdisciplinar (médicos, ortodontistas, fisioterapeu tas,odontopediatras, fonoaudiólogos) 8,26.

A ligação da rinite alérgica e da asma envolve aspectos epidemiológicos, histológicos, fisiopatológicos que merecem ser observados terapeuticamente. Epidemiologicamente essas doenças, freqüentemente, coexistem. No ponto de vista histológico, a mucosa nasal e brônquica têm muitas semelhanças, caracterizada por um mesmo epitélio pseudo-estratificado. Fisiologicamente, elas podem ser relacionadas com o reflexo nasobronquial, ou seja, processos inflamatórios nasais podem provocar uma hiper-responsividade brônquica. Patologicamente pode se observar uma inflamação crônica do epitélio de todo trato respiratório em resposta aos alérgenos através do sistema imunológico. Atualmente a literatura sugere um novo termo para designar esta ligação da rinite alérgica e asma que seria "allergic rhinobronchitis" 5 .

A rinite alérgica é evidenciada como o principal fator etiológico da respiração oral ${ }^{18,22,27}$. A explicação para esse fato é devido ao estabelecimento do processo inflamatório presente nessa morbidade que é desencadeada tanto pelo contato com os alérgenos quanto com agentes irritantes. Esse processo inflamatório desencadeia a obstrução nasal e consequentemente a respiração oral ${ }^{22}$. 
Crianças com rinite alérgica e respiração oral apresentam edema de mucosa nasal, alteração de arcada dentária e alteração do crescimento crânio facial, alteração freqüente na fala, na alimentação, na postura corporal, no aprendizado escolar e no sono ${ }^{27-29}$.

Há um estudo que também referencia a alta prevalência da respiração oral em crianças asmáticas ${ }^{30}$, porém o papel da respiração oral sobre o agravamento da asma era pouco estudado até a pouco tempo. Este estudo relatou que é comum em crianças asmáticas a respiração oral secundária à obstrução nasal ${ }^{30}$.

Em relação à terapêutica, a corticoterapia por inalação nasal, a corticoterapia por inalação oral e a terapia fonoaudiológica são tidas como recurso de primeira linha para pacientes com asma, rinite alérgica e respiração oral ${ }^{31,32}$. A corticoterapia nasal e a inalatória oral diminuem o processo inflamatório das vias aéreas respiratórias, atuando nas mucosas brônquica e nasal ${ }^{31}$. A terapia fonoaudiológica em pacientes alérgicos e respiradores orais tem como objetivo desenvolver a utilização da musculatura facial e corporal de forma efetiva buscando reabilitar a capacidade diafragmática na função respiratória e, ao mesmo tempo, conscientizar a utilização muscular. Essa terapêutica proporciona ao paciente condições de manter a respiração nasal quando não estiver em crise. Por outro lado, a utilização adequada da musculatura é um forte aliado durante as crises. O papel do fonoaudiólogo é de conscientizar o paciente a utilizar a função nasal para que ocorra umidificação do ar, aquecimento e purificação adequados; de trabalhar a propriocepção da respiração, de orientar exercícios para a mobilidade e tonicidade da musculatura orofacial; de adequar as funções estomatognáticas (mastigação, deglutição e fala) e dar mobilidade a musculatura diafragmática com treinos respiratórios em situação controlada e respiração de esforço. Assim, essa terapêutica irá favorecer a adequação da respiração e restabeler a função nasal em pacientes alérgicos e respiradores orais com o intuito de espaçar e reduzir a intensidade dos períodos de agudização da rinite ${ }^{32}$.

Instrumentos para avaliar o impacto da enfermidade crônica no dia a dia dos pacientes, ou seja, sobre a qualidade de vida, já foram desenvolvidos e publicados por investigadores em muitos países. O Health-related quality of live (HRQL), questionário de qualidade de vida que fornece informações sobre o impacto da doença crônica (prejuízos físicos, emocionais e sociais) na vida do paciente. $\mathrm{Na}$ asma, utiliza-se o questionário Paediatric Asthma Quality of life questionnaire (PAQLQ) que identifica os sintomas da asma, o impacto emocional e a limitação social advindas dessa doença ${ }^{33}$.
O questionário mais utilizado na rinite alérgica é o Rhinoconjunctivits quality of life questionnaire (RQLQ) destinado a avaliar a qualidade de vida em adolescentes com rinite alérgica ${ }^{34}$. Não foi encontrado na literatura questionário de qualidade de vida específico para avaliar o impacto da respiração oral.

A inter-relação da asma, da rinite alérgica e da respiração oral refere-se à inflamação alérgica contígua das vias aéreas. Vários autores concordam que o processo inflamatório causada pela rinite alérgica desencadeia a obstrução nasal e, consequentemente, a respiração oral ${ }^{22,27}$. Também citam que os processos inflamatórios nasais podem aumentar ainda mais a hiper-responsividade brônquica e assim intensificar a inflamação crônica do epitélio de todo trato respiratório em resposta aos alérgenos através do sistema imunológico ${ }^{30}$.

Já é evidenciado na literatura que os sintomas da asma, da rinite alérgica e da respiração oral afetam diretamente o bem estar físico, emocional e social do paciente ${ }^{4,6,15-18,23,24}$. Esses fatores prejudicam o sono o que resulta em fadiga e sonolência diurna, contribuindo para redução no aprendizado, na eficiência no trabalho e na diminuição da qualidade de vida ${ }^{35}$.

Esses dados corroboram com a literatura, pois já está registrada a pior qualidade de vida das crianças asmáticas com rinite alérgica em relação às asmáticas sem rinite alérgica, devido aos seus sintomas. É relatado ainda, que a coexistência da asma e da rinite alérgica reflete em diminuição na tolerância aos exercícios e no aumento das faltas nas escolas ${ }^{36}$. E a co-relação da rinite alérgica e da respiração oral apresenta uma alta freqüência de alterações na fala, baixo desempenho escolar e alterações no sono ${ }^{29}$.

Por meio da revisão da literatura realizada foi possível observar a preocupação dos autores em utilizar terapia medicamentosa para aliviar os sintomas da asma e da rinite alérgica, atuando assim na melhoria da qualidade de vida ${ }^{2,12,18,31}$. Fato discordante com os achados de um estudo que citou que o uso dessa terapêutica piora a qualidade do sono, e, consequentemente, provoca sonolência diurna ${ }^{17}$.

Estudos sugerem procedimentos terapêuticos para diminuir o impacto dessas co-morbidades na qualidade de vida ${ }^{32,37}$. Um recurso terapêutico apropriado em casos selecionados é a imunoterapia que pode prevenir o desenvolvimento da asma, reduzir o processo inflamatório e melhorar a qualidade de vida das crianças, especialmente dos sintomas como prurido e obstrução nasal. Com a melhora desses sintomas há uma melhor performance no trabalho o que permite menor uso de drogas. Esse 
fato pode ser observado após 4 meses de imunoterapia ${ }^{37}$. Outro recurso seria o tratamento cirúrgico de pólipo nasal como um fator que implica na diminuição da obstrução nasal, da rinorréia, contribuindo para o desaparecimento da asma e melhoria da função pulmonar. O alívio desta obstrução nasal preserva todo o trato respiratório, diminui a sintomatologia pulmonar, propicia a respiração nasal e favorece uma melhor qualidade de vida ${ }^{38}$.

Medidas não medicamentosas como controle ambiental ${ }^{12}$, tratamento fonoaudiológico ${ }^{23} \mathrm{e}$ medidas para avaliar a qualidade de vida ${ }^{33,34}$ são também relatadas na literatura em favorecer um impacto qualitativo que garantam uma recuperação conjunta no estado físico, emocional e social de crianças e adolescentes.

Entretanto, aumentar quantitativamente a sobrevida dos pacientes através de tratamentos eficazes nem sempre produz esse impacto qualitativo. E, a medida do impacto do tratamento na qualidade de vida do paciente passou a ser observada no momento de selecionar tratamentos mais efetivos e também na implementação de programas de saúde ${ }^{39}$.

Portanto, devido à unicidade das vias aéreas superiores e inferiores, a inter-relação da asma, da rinite alérgica e da respiração oral e o grande impacto dessas co-morbidades na qualidade de vida do indivíduo, profissionais que tratam exclusivamente das vias aéreas inferiores poderiam também considerar, como necessária, a abordagem das vias aéreas superiores e vice-versa ${ }^{5}$. Seria uma abordagem efetuada em equipe interdisciplinar com o objetivo da avaliação global do paciente. Questionários que avaliam a qualidade de vida constituem um recurso propedêutico de grande utilidade e o tratamento não fica limitado ao controle clínico. Existem poucos estudos que verificam o impacto da asma, da rinite alérgica na qualidade de vida do indivíduo, através de questionários específicos, e não foi encontrado estudos de aplicação desse questionário na qualidade de vida do respirador oral. Estudos que avaliam de forma mais precisa esse aspecto devem ser realizados.

\section{CONSIDERAÇÕES FINAIS}

Há consenso na literatura de que existe interrelação dessas co-morbidades.

O impacto da asma, rinite alérgica e respiração oral afetam diretamente a qualidade de vida do indivíduo não só pela alteração respiratória, mas, também pelos prejuízos comportamentais, funcionais e físicos que ocasionam.

O controle dessas morbidades é um tema usual na literatura. Novas propostas terapêuticas englobam o envolvimento de equipes multiprofissionais. Há programas de saúde estruturados com essa visão e objetivam não só melhorar a condição respiratória, mas, também propõem medidas que analisam a qualidade de vida e possibilitam avaliação global do paciente.

\begin{abstract}
Background: quality of life for patient with breathing illnesses Purpose: to review specialized literature focusing on the impact of asthma, allergic rhinitis and mouth breathing on patient's life quality, the relationship between these illnesses and measures that can reduce this impact. Conclusion: it is a consensus in the specialized literature that there is a relationship between these illnesses. Asthma, allergic rhinitis and mouth breathing affect the person's life quality not only producing breathing disorder but also causing behavioral, functional and physical damage. Controlling these illnesses is a usual subject in the specialized literature. New therapeutic proposals include the involvement of multiprofessional teams. There are health programs whose goals are not only the improvement of respiratory condition. They also propose measures that analyze life quality and make possible patient's global evaluation.
\end{abstract}

KEYWORDS: Asthma; Rhinitis; Mouth Breathing; Sickness Impact Profile; Quality of Life

\section{REFERÊNCIAS}

1. Naspitz CK. Epidemiology of allergic respiratory diseases in Brazil. Progr Allergy Clin Immunol. 1997; 4(2):90-3.
2. Bousquet J, Van Cauwenberge P, Khaltaev N, Aria Workshop Group, World Health Organization. Allergic rhinitis and its impact on asthma ARIA workshop report. J Allergy Clin Immunol. 2001; 108(5Suppl):147-334. 
3. Worldwide variations in the prevalence of asthma symptoms. The International study of asthma and allergies in childhood (ISAAC). Eur Respir J. 1998; 12(2):315-35.

4. Fiore RW, Comparsi AB, Reck CL, Oliveira $\mathrm{JK}$, Pampanelli KB, Fritscher CC. Variação na prevalência de asma e atopia em um grupo de escolares de Porto Alegre, Rio Grande do Sul. J Pneumol. 2001; 27(5):237-42.

5. Simons FER. Allergic rhinobrochitis: the asthmaallergic rhinitis link. J Allergy Clin Immunol. 1999; 104:534-40.

6. Queluz DP, Gimenes CMM. A síndrome do respirador bucal. Rev CROMG. 2000; 6(1):4-9.

7. Breda D, Moreira HSB. Avaliação postural e da função respiratória em crianças com rinite alérgica, hipertrofia de adenóide e síndrome do respirador bucal. Fisioter Bras. 2003; 4:247-52.

8. Marchesan IQ. A equipe de trabalho no respirador oral. In: Krakauer LH, Francesco R, Marchesan $I Q$, organizador. Respiração oral. São José dos Campos: Pulso; 2003. p.163-7.

9. Blaiss M. Measuring outcomes in pediatric asthma. Allergy Asthma Proc. 2001; 22:63-5.

10. Greenberger PA. Therapy in the management of the rhinitis/asthma complex. Allergy Asthma Proc. 2003; 24(6):403-7.

11. Fontes MJF. Asma brônquica e síndrome sibilante: descrição e avaliação de uma proposta de tratamento do serviço público de saúde em parceria com a Universidade Federal de Minas Gerais a partir de dados coletivos e de um grupo de crianças [tese]. Belo Horizonte (MG): Universidade Federal de Minas Gerais; 2002.

12. Hetzel JL, Silva LCC. Tratamento atual da asma. In: Porto NS, Araújo E, Godoy DV, organizador. Doenças da vias aéreas: uma visão clínica integradora (viaerologia). Rio de Janeiro: Revinter; 2001.p.267-76.

13. La Scala CSK, Naspitz CK, Solé D. Adaptação e validação do Pediatric Asthma Quality of Life Questionnaire (PAQLQ-A) em crianças e adolescentes brasileiros com asma. J Pediatr. 2005; 81(1):54-60.

14. Juniper EF, Guyatt GH, Feeny DH, Ferrie PJ, Griffith LE, Townsend M. Measuring quality of life in children with asthma. Qual Life Res.1996; 5(1):35-46.

15. Schmier JK, Chan KS, Leidy NK. The impact of asthma on health-related quality of life. J Asthma. 1998; 35(7):585-97.

16. $\mathrm{Ng} \mathrm{ML,} \mathrm{Warlow} \mathrm{RS,} \mathrm{Chrishanthan} \mathrm{N,} \mathrm{Ellis}$ C, Walls R. Premiliary criteria for the definition of allergic rhinitis: a systematic evalution of clinical parameters in a diseasse cohort (I). Clin Exp Allergy. 2000; 30(9):1314-31.
17. Balbani APS, Caniello M, Miyake MAM, Mello Júnior JR, Butugan O. Rinites e anti-histamínicos: impacto na cognição e psicomotricidade. Rev Bras Alerg Imunopatol. 2001; 24(3):106-14.

18. Craig TJ, Hanks CD, Fisher LH. How do topical nasal corticosteroids improve sleep and daytime somnolence in allergic rhinitis? J Allerg Clin Immunol. 2005; 116(6):1264-6.

19. Blaiss MS, Allergic Rhinits in Schoolchildren Consensus Group. Allergic rhinitis and impairment issues in schoolchildren: a consensus report. Curr Med Res Opin. 2004; 20(12):1937-52.

20. Blaiss MS. Important aspects in management of allergic rhinitis: compliance, cost, and quality of life. Allergy Asthma Proc. 2003; 24(4):231-8.

21. Rodrigues HOSN, Faria SR, Paula FSG, Motta AR. Ocorrência de respiração oral e alterações miofuncionais orofaciais em sujeitos em tratamento ortodôntico. Rev CEFAC. 2005; 7(3):356-62.

22. Cintra CFSC, Castro FFM, Cintra PPVC. As alterações oro-faciais apresentadas em pacientes respiradores bucais. Rev Bras Aler Imunopatol. 2000; 23(2):78-83.

23. Motonaga SM, Berth LC, Lima A. Respiração bucal: causas e alterações no sistema estomatognático [dissertação]. Ribeirão Preto (SP): Faculdade de Medicina de Ribeirão Preto; 2004.

24. Justiniano JR. Respiração bucal. J Bras Ortond Ortop Max. 1996; 1(1):44-6.

25. Vera CFD, Conde GES, Wajnsztejn R, Nemr K. Transtornos de aprendizagem e presença de respiração oral em indivíduos com diagnóstico de transtornos de déficit de atenção/hiperatividade (TDAH). Rev CEFAC. 2006; 8(4):441-55.

26. Junqueira $P$, Parro FM, Toledo MR, Araújo RLT, Di Francesco R, Rizzo MC. Conduta fonoaudiológica para pacientes com diagnóstico de rinite alérgica: relato de caso. Rev CEFAC. 2005; 7(3):336-9.

27. Barros JRC, Becker HMG, Pinto JA. Avaliação de atopia em crianças respiradoras bucais atendidas em centro de referência. J Pediatr. 2006; 82(6):458-64.

28. Branco A, Ferrari GF, Weber AS. Alterações orofaciais em doenças alérgicas de vias aéreas. Rev Paul Pediatr. 2007; 25(3):266-70.

29. Rizzo C, Hauache S, Naspitz N, Pignatari S, Junqueira $P$, Hallinan $M$, Weckx L, Naspitz C. Characteristics of children with allergic rhinitis and chronic mouth breathing. J Allergy Clin Immunol. 2002; 109(1):S263.

30. Venetikidou A. Incidence of malocclusion in asthmatic children. J Clin Pediatr Dent. 1993; 17(2):89-94.

31. Camargos PA, Rodrigues ME, Lasmar LM. Simultaneous treatment of asthma and allergic rhinitis. Pediatr Pulmonol. 2004; 38(3):186-92. 
32. Rahal A, Krakauer LH. Avaliação e terapia fonoaudiológica com respiradores bucais. Rev Dental Press Ortop Facial. 2001; 6(1):83-6.

33. Juniper EF. How important is quality of life in pediatric asthma? Pediatr Pneumol. 1998; 24(S15):17-21.

34. Silva MGN, Adaptação e validação do questionário "RQLQ" para avaliação da qualidade de vida em crianças e adolescentes com rinite alérgica [tese]. São Paulo (SP): Universidade Federal de São Paulo; 1999.

35. Ferguson BJ. Influences of allergic rhinitis on sleep. Otolaryngol Head Neck Surg. 2004; 130(5):617-29.

36. Pérez Lu JE, Huamán JC, Ortigosa PRC, Pérez Lu LE, Jaramillo MG. Características de niños asmáticos con rinitis en el Hospital Nacional Cayetano Heredia. Diagnóstico. 2004; 43(2):65-70.

37. Moncayo Coello CV, Rosas Vargas MA, Del Rio Navarro BE, Lerma Ortiz L, Velzquez Armenta Y, Sienra Monge JJ. Quality of life in children with allergic rhinitis before and after being treated with specific immunotherapy (cases end controls). Rev Alerg Mex. 2003; 50(5):170-5.

38. Triglia JM, Nicollas R. Nasal and sinus polyposis in children. Laryngoscope. 1997; 107(7):963-6.

39. Ramos-Cerqueira ATA, Crepaldi AL. Qualidade de vida em doenças pulmonares crônicas: aspectos conceituais e metodológicos. J Pneumol. 2002; 26(4):207-13. 